\title{
Multiple skeletal lesions and pleural effusion owing to Histoplasma capsulatum infection in an immunocompetent patient from a non- endemic region
}

\author{
F E Suleman, MB ChB, FCRad (Diag) SA, M Med Rad (Diag) \\ Department of Imaging and Diagnostic Radiology, University of Limpopo (Medunsa Campus), Pretoria
}

\section{P A Scheepers, MB ChB, FCRad (Diag) SA, M Med Rad (Diag)}

Department of Imaging and Diagnostic Radiology, University of Limpopo (Medunsa Campus); consultant radiologist in private practice

Corresponding author: F Suleman (tar@absamail.co.za)

\begin{abstract}
We describe a rare case of multiple skeletal lesions and a pleural effusion owing to Histoplasma capsulatum infection in a 16-year-old immunocompetent girl residing in a non-endemic region. Of note is that she had a lesion within a thoracic vertebra. Following an extensive literature search, we found that vertebral involvement by $H$. capsulatum had not been previously described. Pleural effusion and skeletal lesions are both rare manifestations of $H$. capsulatum infection.
\end{abstract}

\section{Case report}

A 16-year-old girl from Venda presented with a 3-month history of gradual onset of weakness involving both legs as well as bilateral knee pain. She was otherwise well. On clinical examination, she was not acutely ill, but had paraparesis and loss of sphincter control. No skin lesions were noted. Blood test results showed an ESR of $70 \mathrm{~mm} / \mathrm{hr}$ and a mildly raised WCC. HIV tests were not performed because the patient was a minor and the guardians were not available for consent. However, the CD4 count was found to be $>900$ cells $/ \mathrm{mm}^{3}$.

Radiographs of the spine, chest and both knees were obtained. These showed a pleural effusion (Fig. 1) and multiple lytic lesions within the metadiaphyses of the long bones around the knees (Fig. 2) as well as within the fourth thoracic vertebral body.

An urgent MRI of the spine showed abnormal T1 hypo- and T2 hyperintensity within the body and posterior elements of T4. Abnormal enhancement was demonstrated with post-contrast administration (Figs 3 - 5). An associated extradural soft-tissue mass caused compression of the spinal cord. Nuclear medicine studies (MDP bone scan) demonstrated increased uptake in all lesions and in addition showed abnormal uptake in both proximal humeri (Fig. 6).

The involved vertebra and surrounding soft-tissue mass were surgically removed and spinal fusion was performed. The excised tissue was sent for histological investigation, which revealed a chronic inflammatory infiltrate composed of lymphocytes, plasma cells and numerous foam cells (Fig. 7). Special staining techniques (periodic acid Schiff and silver stain) showed the presence of multiple yeast cells both within and outside the foamy histiocytes (Figs 8 and 9). Some yeast cells demonstrated budding. Morphologically, the fungus was consistent with
Histoplasma capsulatum. Accordingly, a diagnosis of disseminated $H$. capsulatum infection was made.

\section{Discussion}

The literature describes two distinct clinical forms of histoplasmosis that have different geographical distributions worldwide. African histoplasmosis is attributed to $H$. duboisii infection which most commonly occurs between the Sahara and Kalahari Deserts. The most common site of infection is the skin but it can also involve bone, with skeletal manifestations occurring in up to two-thirds of patients with disseminated infection. ${ }^{1}$ The femur is the most frequently affected skeletal site, with diaphyseal involvement in young children and juxtametaphyseal involvement in older children and adults. ${ }^{1,2}$ Skeletal lesions are usually multiple, and spinal involvement resulting in paraplegia has been reported. ${ }^{3}$

H. capsulatum infection is responsible for American or classical histoplasmosis infection. It has a worldwide distribution but is most commonly found in certain parts of North and Central America. The reasons for this endemic distribution are unknown but are thought to include climate, humidity and soil characteristics. Growth of the organism is enhanced by bird and bat excrement in the soil, so a typical exposure history would be that of cave exploration. ${ }^{4}$ Our patient gave no history of entering caves. However, further enquiry

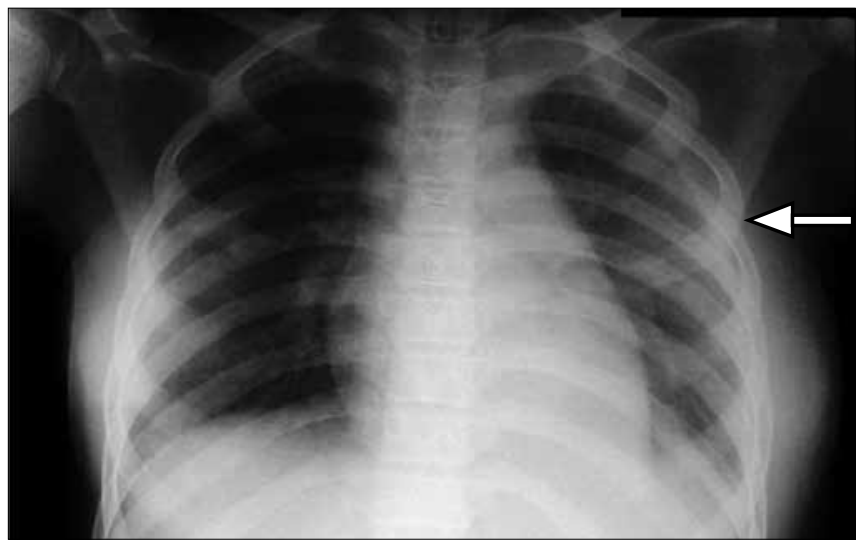

Fig. 1. Supine chest radiograph of the patient, with a large pleural effusion on the left (arrow). 


\section{CASE REPORT}
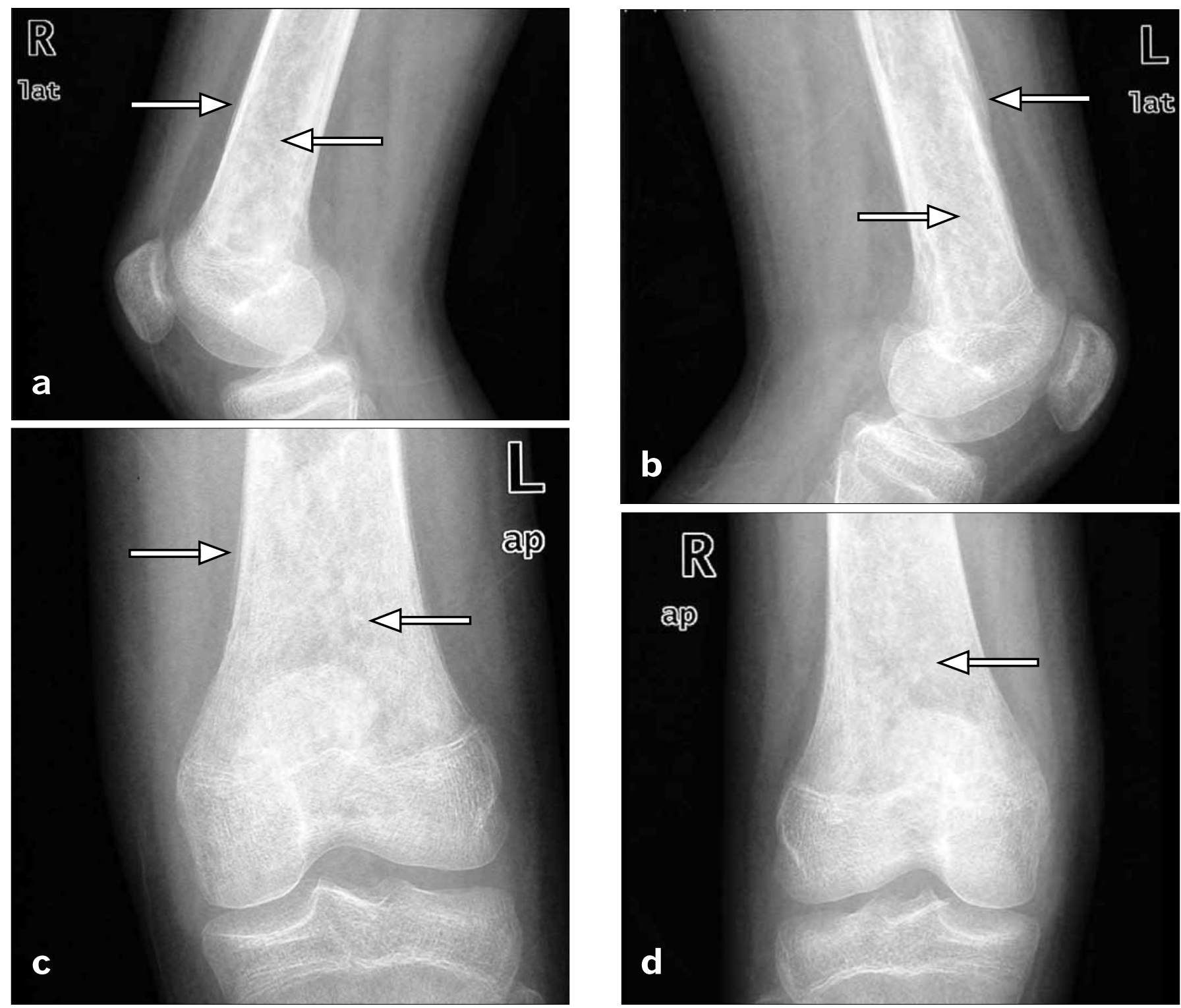

Fig. 2. Lateral $(2 a, 2 b)$ and antero-posterior $(2 c, 2 d) X$-rays of both knees show permeative lesions of the metadiaphyses of both distal femurs, with a solid smooth periosteal reaction on the left femur.

revealed that she resides in a rural area surrounded by caves inhabited by bats.

Three types of clinical disease may occur in patients with histoplasmosis. The most common presentation is acute pulmonary histoplasmosis. ${ }^{5}$ Patients typically present with pulmonary symptoms that run a self-limiting course. Chest radiographs may show hilar lymphadenopathy and upper lobe predominant nodules. Chronic pulmonary histoplasmosis resembles tuberculosis. ${ }^{4}$ Some patients may go on to disseminated infection with widespread disease, which can be fatal in the absence of therapy. Even in this setting, pleural effusion and skeletal involvement is extremely rare. ${ }^{4}$

The distal femoral lesions found in our patient resemble those frequently seen in $H$. duboisii infection, which typically show juxtametaphyseal destruction of bony trabeculae. Owing to the presence of multiple bone lesions, a provisional diagnosis of chronic multifocal osteomyelitis was considered, but histological analysis revealed the presence of multiple yeast cells both within and outside foamy histiocytes that morphologically resembled $H$. capsulatum. We could find only 8 cases of bony lesions due to H. capsulatum in the literature; to the best of our knowledge, the present one would be the first reported case of a patient with vertebral body involvement. ${ }^{6}$

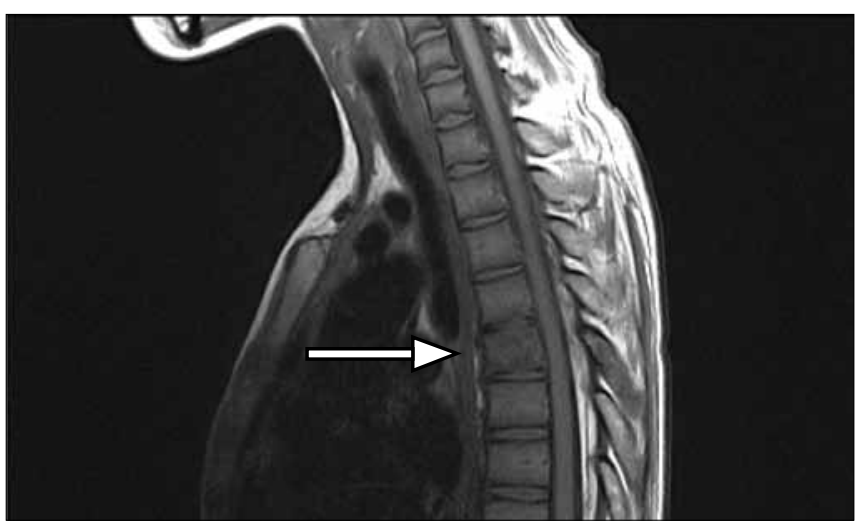

Fig.3. T1-weighted MRI shows low intensity changes of the T4 vertebra (arrow) and involvement of the inferior endplate of the vertebral body of T3. 


\section{CASE REPORT}

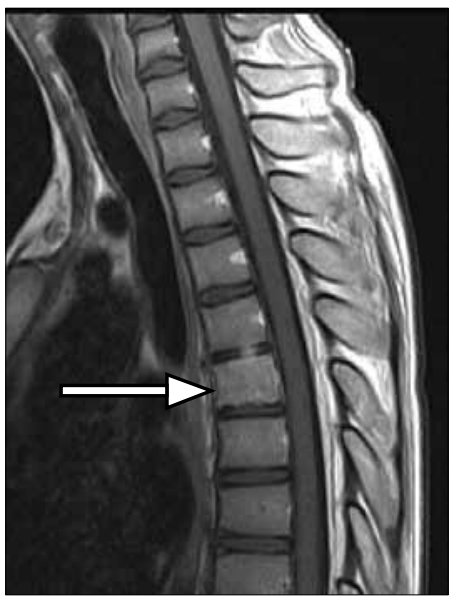

Fig. 4. Post contrast T1-weighted MRI shows slight enhancement of T4 (arrow) and the T3-T4 disc.

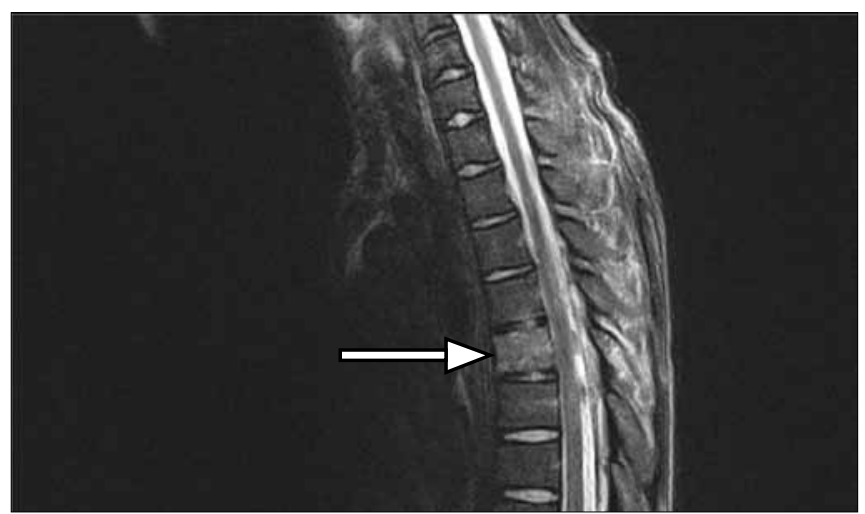

Fig. 5. T2-weighted MRI shows high signal intensity changes of the T4 (arrow) vertebra. The adjacent disc spaces are narrowed and show loss of the normal signal intensity. There is high signal change within the cord, and the outline is irregular and ill-defined. There are high signal changes in the posterior elements and surrounding soft tissue.

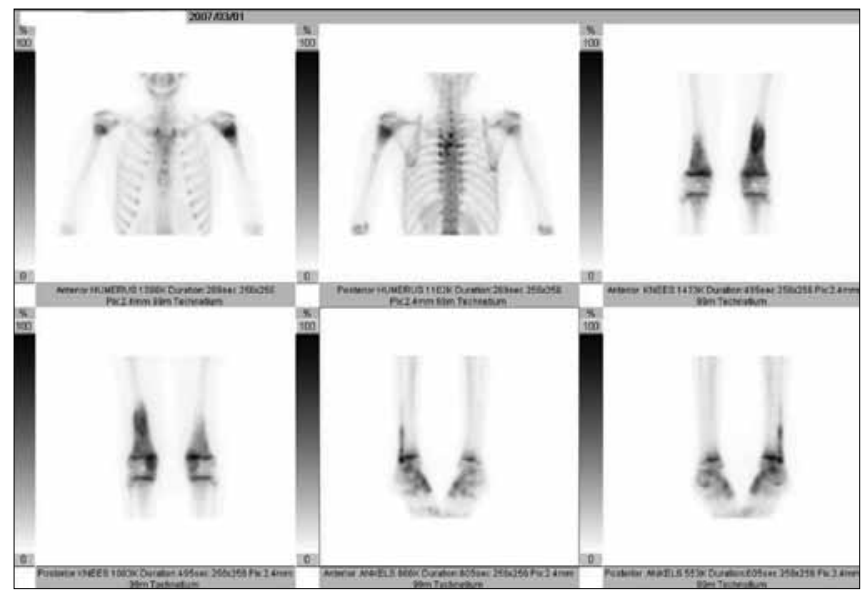

Fig. 6. MDP bone scan shows uptake in the proximal humeri, distal femurs and the $T 3$ and $T 4$ vertebral bodies.

\section{Conclusion}

This is an unusual case of chronic disseminated $H$. capsulatum infection, having occurred outside an endemic region and with the patient immunocompetent. She presented with two unusual clinical

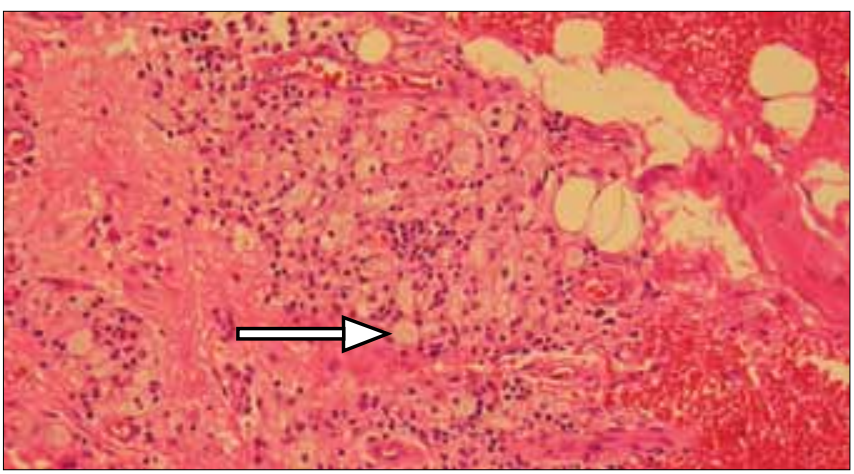

Fig 7. Haematoxylin and eosin stain showing mixed chronic inflammation with foam cells (arrow).

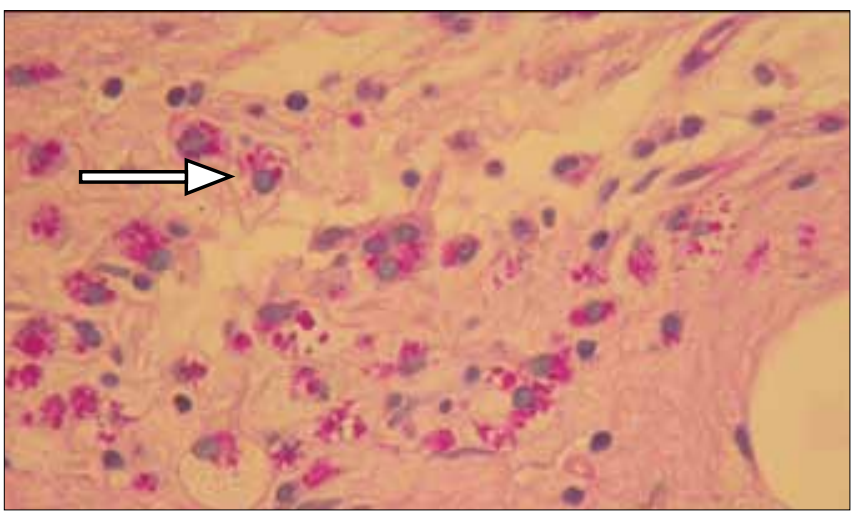

Fig 8. Periodic acid Schiff's stain showing intracytoplasmic yeast cells (arrow).

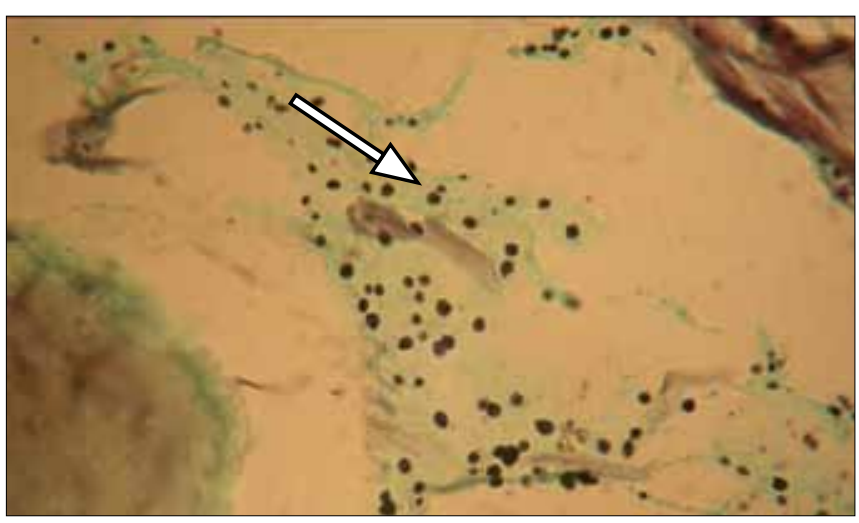

Fig 9. Silver stain demonstrating yeast cells with faint halo (arrow).

manifestations, i.e. multiple bone lesions, including a vertebral body lesion, as well as a left-sided pleural effusion. None of the radiographic findings raised any suspicion of $H$. capsulatum infection, and it was thanks to the astute histopathologist that the diagnosis was made. As a result, the patient went on to receive appropriate treatment.

1. Thompson EM, Ellert J, Peters LLW, Adjukiewicz A, Mabey D. Histoplasma duboisii infection of bone. Br J Radiol 1981;54:518-521.

2. Onwuasoigwe O, Gugnani HC. African histoplasmosis: osteomyelitis of the radius. Mycoses 1998;41:105107.

3. Cockshott WP, Lucas AO. Radiological findings in Histoplasmosis duboisii infections. $\mathrm{Br} J$ Radiol 1964;37:653-660

4. Aziz R, Khan N, Qayum I, Khan AR. Histoplasmosis - case report. J Ayub Med Coll Abbottabad 2002;14(4):42-44

5. Kapotsis GE, Daniil Z, Malagari K, et al. A young male with chest pain, cough and fever. Eur Respir J 2004;24:506-509.

6. Jones RC, Goodwin Jr RA. Histoplasmosis of bone. Am J Med 1981;70:864-866. 